\title{
Successful closure of large blunt macular chorioretinal rupture: a case report
}

This article was published in the following Dove Press journal:

Clinical Ophthalmology

25 February 2012

Number of times this article has been viewed

\section{Daisuke Muramatsu' \\ Takuya Iwasaki \\ Tsuyoshi Agawa' \\ Masahiko Usui' \\ Hiroshi Goto' \\ 'Department of Ophthalmology, Tokyo \\ Medical University, ${ }^{2}$ Department of Ophthalmology, Tokyo Medical \\ University lbaraki Medical Center, \\ Tokyo, Japan}

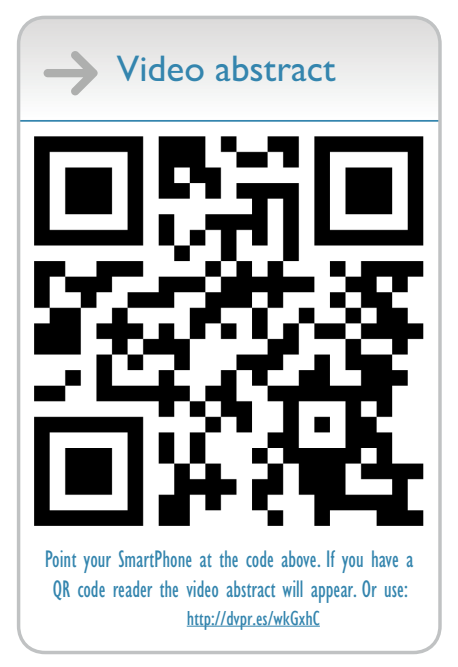

Correspondence: Daisuke Muramatsu Department of Ophthalmology, Tokyo Medical University, 6-7-I Nishishinjuku, Shinjuku-ku, Tokyo 160-0023, Japan

$\mathrm{Tel}+8 \mathrm{I} 333426 \mathrm{II}$ I ext 5880

Fax +8I 333469170

Email gmura08I5@yahoo.co.jp

\begin{abstract}
The authors present a rare case of large chorioretinal rupture caused by blunt traumatic injury of the globe. A 22-year-old woman sustained a blunt injury to her left eye. The best-corrected Snellen visual acuity was $2 / 20$ in her left eye, and hyphema and vitreous hemorrhage were noted. The day after the injury occurred the vitreous hemorrhage had disappeared. Fundus examination revealed a crescent-shaped retinal rupture three disc diameters in size near the macula, and a choroidal rupture six disc diameters in size that was over the vascular arcade. Three days after the injury, vitrectomy with internal limiting membrane peeling was performed. Postoperative prone positioning was maintained for 4 days. Five days postoperatively, closure of the ruptured retina was confirmed. The visual acuity improved to 16/20 4 months after surgery and this was maintained over a 48-month period. In conclusion, early vitrectomy with internal limiting membrane peeling after injury was effective for a case involving severe blunt chorioretinal rupture with closed globe injury.
\end{abstract}

Keywords: choroidal rupture, retinal rupture, traumatic macular hole, closed globe injury, internal limiting membrane

\section{Introduction}

Traumatic macular holes can have various clinical courses: some close spontaneously, while others persist without treatment. ${ }^{1}$ Recent papers have reported the efficacy of vitrectomy for treatment of traumatic macular hole. ${ }^{2,3}$ However, the role of vitrectomy is not well established. Recent studies have shown a higher closure rate of macular holes with internal limiting membrane (ILM) peeling than with the conventional method. ${ }^{4-6}$ Ocular blunt trauma sometimes results in not only a macular hole but also choroidal rupture. The choice of treatment is more controversial in such cases than when there is a macular hole only.

The authors present a rare case of severe chorioretinal rupture with closed globe injury that achieved a good outcome through early vitrectomy with ILM peeling.

\section{Case report}

A 22-year-old woman was hit in her left eye with a beer bottle. Initially, when she visited the emergency room, the eyelids were swollen, and the Snellen visual acuity (VA) was 30/20 in the right eye and 3/100 (2/20 when wearing -6.5 dioptors) in the left eye, due to injury-induced high myopia, hyphema, and vitreous hemorrhage. Fundus examination was impossible because of intraocular hemorrhage. One day after the injury the vitreous hemorrhage had resolved. Ophthalmoscopic examination revealed both a crescent-shaped retinal rupture near the macula three disc diameters (DDs) in 
size and a choroidal rupture six DDs in size that was over the vascular arcade. Subretinal hemorrhage near the disc, limited retinal detachment in the temporal fundus of the disc, and peripheral retinal edema were observed (Figure 1A and B). The best-corrected VA had decreased to 9/100. Fluorescein angiography revealed a window defect of the ruptured area, leakage in the temporal retina near the macula, and blocked fluorescence. Goldmann perimetry revealed expansion of Marriott scotoma, a crescent-shaped scotoma within $20^{\circ}$, and a nasal peripheral visual field defect. Optical coherence tomography revealed rupture and subsidence of the retina and choroid, and a retinal detachment (Figure 1C).

The patient underwent 20-gauge pars plana vitrectomy 3 days after the injury. After removing the vitreous core,

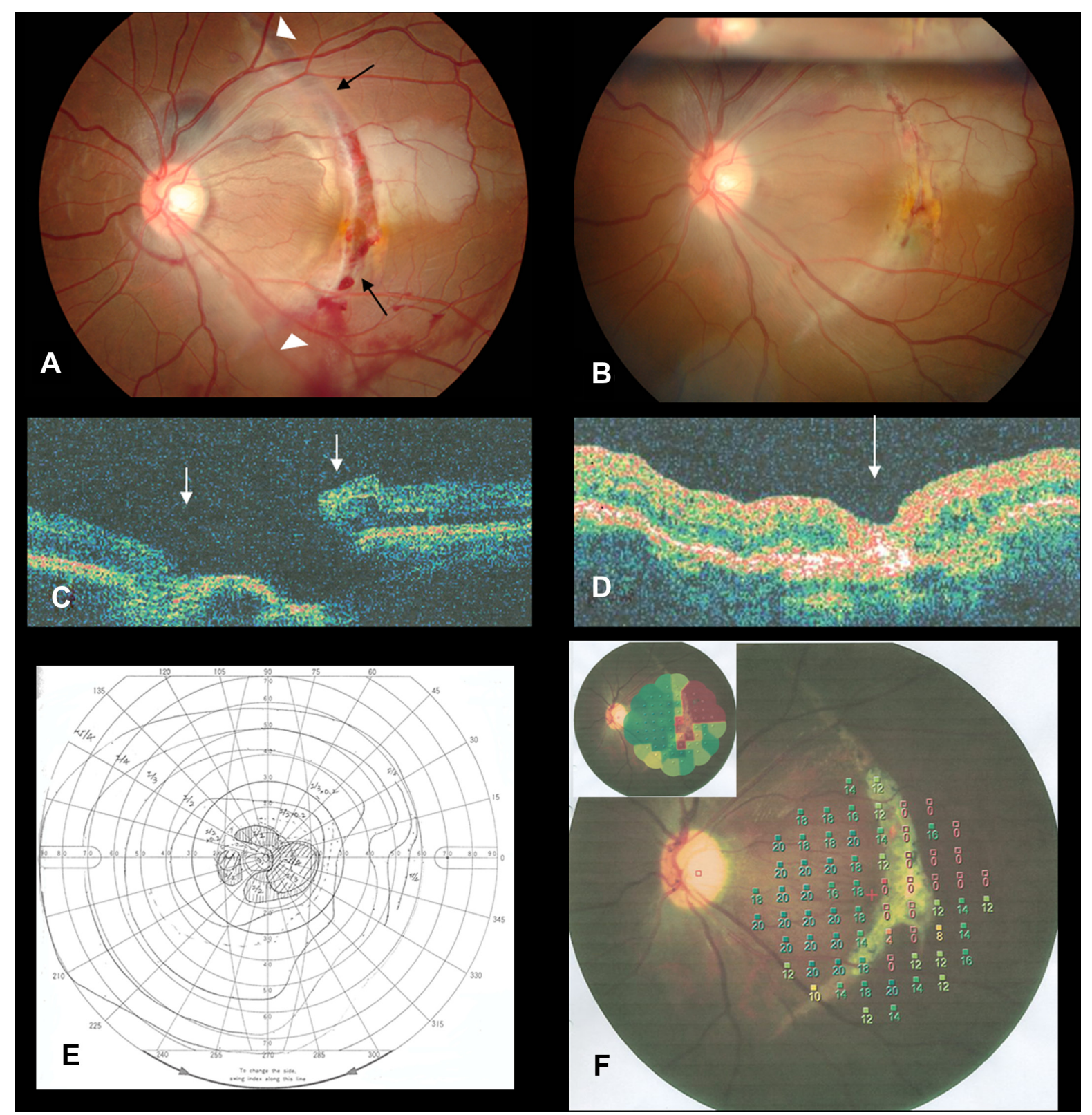

Figure I (A) Preoperative fundus photograph: a crescent-shaped retinal rupture near the macula (indicated by the black arrows), a choroidal rupture six disc diameters in size (indicated by the white arrowheads), subretinal hemorrhage, and peripheral retinal edema are seen. (B) Fundus photograph 7 days after surgery: the ruptured retina is closed. (C) Preoperative optical coherence tomography: rupture and subsidence of the retina and choroid and retinal detachment were identified (the arrows indicate the edges of the ruptured retina). (D) Postoperative optical coherence tomography: closure of the ruptured retina is seen, but the choroid and retina show subsidence, and the retinal pigment epithelium (RPE) layer has become thickened (the arrow indicates the scarred RPE near the macula). (E) Goldmann perimetry 4 months after surgery: the central scotoma is decreased, but the nasal crescent-shaped scotoma has become slightly bigger. (F) MP-I micoperimetry: the ruptured choroid and RPE show scarring; retinal sensitivity is obtained; the ruptured area and superior temporal region of the macula have $0 \mathrm{~dB}$ sensitivity, but MP-I micoperimetry shows the fixation point at the nasal macula; the nasal retina of macular area has normal retinal sensitivity of 16-20 dB; inferior temporal retina shows retinal sensitivity of 6-12 dB. 
a posterior vitreous detachment was created, followed by ILM peeling over the vascular arcade using indocyanine green staining. The remaining vitreous, including the vitreous base, was removed. Fluid-air exchange was performed, followed by filling the eye with $20 \%$ sulfur hexafluoride gas. Phacoemulsification was not performed.

Postoperative prone positioning was maintained for 4 days. Five days postoperatively, closure of the ruptured retina was confirmed (Figure 1B). Seven days after surgery, the corrected VA was 8/20.

Four months after surgery, VA had improved to $12 / 20$ when wearing -2.5 diopters, and myopia was decreased. The ruptured choroid and retinal pigment epithelium (RPE) showed gradual scarring. Fluorescein angiography revealed late-phase staining at the site of the retinal rupture and a window defect at the site of the choroidal rupture. Optical coherence tomography revealed closure of the ruptured retina, but the choroid and retina showed subsidence, and the RPE layer had thickened (Figure 1D). Goldmann perimetry revealed a decrease of the central scotoma, but the nasal crescentshaped scotoma had become slightly larger. Microperimetry (MP-1 microperimeter; Nidek Co, Ltd, Aichi, Japan) revealed that retinal sensitivity at the ruptured area and superior temporal region of the macula was $0 \mathrm{~dB}$; however, the fixation point at the nasal region of the macula was detected with the MP-1 microperimeter (Figure 1E and F).

VA had improved to $16 / 20$ when wearing -1.0 diopters at 12 months post injury, and visual function was maintained for 48 months postoperatively.

\section{Discussion}

There have been many reports of vitrectomy for traumatic macular hole. Recently, ILM peeling has been performed to increase the success rate of vitrectomy. In general, traumatic macular holes are small in size. To the best of the authors' knowledge, there have been no reports on the treatment of large retinal ruptures caused by blunt trauma. Observation without treatment for 1-3 months following injury is the normal course for small traumatic macular holes, as spontaneous closure of traumatic macular holes is common. However, in the current case, the retinal rupture was three DDs in size and the choroidal rupture was six DDs. The authors considered that vitreous tamponade alone would not be able to close the retinal break, and therefore chose to perform vitrectomy soon after the injury.

The purposes of vitrectomy were to remove the vitreous traction to close the retinal break and to permit gas tamponade. The width of the retinal break was large, one DD, so the ILM was removed to decrease tangential traction.

Although, this case presented a severe retinal rupture and a choroidal rupture, favorable VA of 16/20 was acquired. Yeung et $\mathrm{al}^{7}$ reported a closed globe injury with choroidal rupture, but final VA in their case was poor: counting fingers to 20/60. Miyake and $\mathrm{Ando}^{8}$ reported that the visual outcome was poor unless vitrectomy was performed within 2 months of injury.

\section{Conclusion}

The good outcome in this case might have been because of the early surgical intervention to prevent scarring at the macula, and peeling the ILM to remove tractional force and cell proliferation. Additionally, it was fortunate that the RPE atrophy was spared at the macular area. However, some visual field defect remained, because the nerve fiber layer was disrupted. The MP-1 microperimeter was very useful in this case for checking and following the retinal sensitivity. As there have been reports of choroidal neovascularization after choroidal rupture, long-term follow-up is required. The results suggest that vitrectomy with ILM peeling early after injury can be considered for severe blunt chorioretinal rupture.

\section{Acknowledgment}

The authors would like to thank Sean Hackett MSc for his assistance.

\section{Disclosure}

The authors report no conflicts of interest in this work. No financial support was received for this submission.

\section{References}

1. Yamada H, Sakai A, Yamada E, Nishimura T, Matsumura M. Spontaneous closure of traumatic macular hole. Am J Ophthalmol. 2002;134(3): 340-347.

2. Amari F, Ogino N, Matsumura M, Negi A, Yoshimura N. Vitreous surgery for traumatic macular holes. Retina. 1999;19(5):410-413.

3. García-Arumí J, Corcostegui B, Cavero L, Sararols L. The role of vitreoretinal surgery in the treatment of posttraumatic macular hole. Retina. 1997;17(5):372-377.

4. Hirata A, Tanihara H. Ruptured internal limiting membrane associated with blunt trauma revealed by indocyanine green staining. Graefes Arch Clin Exp Ophthalmol. 2004;242(6):527-530.

5. Ikeda T, Sato K, Otani H, Yu S, Ikeda N, Mimura O. Vitreous surgery combined with internal limiting membrane peeling for traumatic macular hole with severe retinal folds. Acta Ophthalmol Scand. 2002;80(1): 88-90.

6. Kuhn F, Morris R, Mester V, Witherspoon CD. Internal limiting membrane removal for traumatic macular holes. Ophthalmic Surg Lasers. 2001;32(4):308-315. 
7. Yeung L, Chen TL, Kuo YH, et al. Severe vitreous hemorrhage associated with closed-globe injury. Graefes Arch Clin Exp Ophthalmol. 2006; 244(1):52-57.
8. Miyake Y, Ando F. Surgical results of vitrectomy in ocular trauma. Retina. 1983;3(4):265-268.

\section{Publish your work in this journal}

Clinical Ophthalmology is an international, peer-reviewed journal covering all subspecialties within ophthalmology. Key topics include: Optometry; Visual science; Pharmacology and drug therapy in eye diseases; Basic Sciences; Primary and Secondary eye care; Patient Safety and Quality of Care Improvements. This journal is indexed on
PubMed Central and CAS, and is the official journal of The Society of Clinical Ophthalmology (SCO). The manuscript management system is completely online and includes a very quick and fair peer-review system, which is all easy to use. Visit http://www.dovepress.com/ testimonials.php to read real quotes from published authors. 